\title{
EVALUACIÓN DEL ENVEJECIMIENTO EN PERSONAS CON DISCAPACIDAD INTELECTUAL MEDIANTE UNA PRUEBA DE SCREENING CONDUCTUAL
}

\author{
Raquel Braga Santos \\ Asociación Juan XXIII, C/ Eduardo Blanco Amor, Pontevedra \\ bragasantos.raquel@gmail.com \\ Elena Felipe-Castaño \\ Universidad de Extremadura. Departamento de Psicología y Antropología. Cáceres. efelipe@unex.es
}

Recepción Artículo: 15 mayo 2021

Admisión Evaluación: 15 mayo 2021

Informe Evaluador 1: 19 mayo 2021

Informe Evaluador 2: 24 mayo 2021

Aprobación Publicación: 01 junio 2021

\section{AGRADECIMIENTOS}

Este artículo ha sido publicado con el apoyo de la Ayuda de consolidación de Grupos de investigación (Junta de Extremadura GR18091). Las autoras quieren agradecer su apoyo.

\section{RESUMEN}

El incremento de la esperanza de vida en personas con discapacidad intelectual (PDI) está teniendo un gran impacto en sus necesidades de atención y en los recursos asociados. Las PDI necesitan mantener su calidad de vida en la vejez y para ello es necesario poder detectar precozmente la aparición de un envejecimiento patológico y así establecer apoyos de manera especializada e individualizada. Contar con pruebas de cribado que permitan a los profesionales de atención directa detectar de forma temprana estos indicadores y así derivarlos a los servicios especializados, es fundamental. El objetivo de este trabajo fue describir las características de una prueba de screening conductual de 31 ítems, con un formato de respuesta de cuatro categorías, diseñada para el registro de conductas asociadas al envejecimiento. La Escala fue administrada por profesionales de atención directa a 64 PDI de edades comprendidas entre los 35 y 65 años. Se cumplió con las normas éticas de la Declaración de Helsinki. La escala permite describir las conductas asociadas a los procesos psicológicos básicos, las actividades de la vida diaria (AVD) y el estado emocional, que aparecen más frecuentemente en PDI. Se puede considerar una herramienta útil para los profesionales de atención directa. No obstante es necesario profundizar en los análisis de fiabilidad y validez predictiva que confirmen su utilidad en la detección precoz de trastornos asociados al envejecimiento patológico y patologías relacionadas con la salud mental. En futuras investigaciones pretendemos profundizar en estos aspectos, pues consideramos que disponer de herramientas de evaluación fiables y válidas, 


\section{EVALUACIÓN DEL ENVEJECIMIENTO EN PERSONAS CON DISCAPACIDAD INTELECTUAL MEDIANTE UNA PRUEBA DE SCREENING CONDUCTUAL}

facilitan la detección temprana, y permiten implementar programas de prevención que mejoran la atención a la salud mental en PDI en proceso de envejecimiento.

Palabras clave: discapacidad intelectual; screening; envejecimiento; atención directa

\section{ABSTRACT}

Assessment of aging in people with intellectual disabilities using a behavioral screening

test. The increase in life expectancy in people with intellectual disabilities (IDPS) is having a great impact on their care needs and associated resources. IDPs need to maintain their quality of life in old age and for this, assessment and diagnosis services must be able to detect early the appearance of pathological aging and thus establish specialized and individualized support. It is essential to have screening tests, which allow direct care professionals to detect these indicators early and thus refer them to specialized services. The objective of this work was to describe the characteristics of a 31-item behavioural screening test, with a response format of four categories, designed to record behaviours associated with aging. The Scale was administered by direct care professionals to 64 IDPs aged between 35 and 65 years. The ethical standards of the Declaration of Helsinki were complied. The scale allows describing the behaviours associated with basic psychological processes, activities of daily living (ADL) and emotional state, which appear more frequently in PDI. It can be considered a useful tool for direct care professionals. It would be necessary to deepen in the analyses of reliability and predictive validity that confirm its usefulness in the early detection of disorders associated with pathological aging and pathologies related to mental health. In future research, we intend to delve into these aspects, as we consider that having reliable and valid assessment tools facilitate early detection, and allow the implementation of prevention programs that improve care for mental health pathologies in PDIs who are ageing.

Keywords: intellectual disability; screening; ageing; direct attention

\section{INTRODUCCIÓN}

A lo largo de la última década, el interés por la investigación y la aparición consecuente de publicaciones sobre envejecimiento en personas con discapacidad intelectual (PDI), ha ido aumentando de forma progresiva. La investigación ha ido ofreciendo respuestas a una nueva realidad, que es el aumento de la esperanza de vida de las PDI, que se ha ido produciendo en la última mitad de siglo, y con ello la aparición de nuevas necesidades asociadas a la edad, con gran impacto en la calidad de vida de estas personas (Del Campo, Arias, Fernández \& Castro, 2007).

En 2007, FEAPS ya propuso la necesidad de creación de recursos específicos para personas mayores con discapacidad intelectual o bien la adecuación y adaptación de los ya existentes, pues los modelos de atención, carecían en ese momento de consideraciones metodológicas específicas para una población envejecida y los equipos de evaluación no poseían las herramientas necesarias y adecuadas para llevar a cabo diagnósticos fiables.

No obstante, a pesar de este mayor interés, la investigación sigue siendo insuficiente, y en la actualidad, los instrumentos de evaluación específicos para población con discapacidad intelectual son muy escasos en España en cualquier rango de edad. Esto es debido, en parte, a que el proceso de evaluación en personas con discapacidad intelectual presenta ciertas particularidades que hace que sea un proceso complejo (García-Alba, EstebaCastillo, \& Viñas-Jornet, 2018).

La discapacidad intelectual tiene como principal característica las limitaciones en el funcionamiento intelectual, así como en las habilidades conceptuales y prácticas de la vida diaria (APA, 2013), lo que supone un reto importante para la adaptación de pruebas diagnósticas, aunque no es el único. A grandes rasgos, podemos decir que a mayores limitaciones, habría una mayor complejidad en la adaptación de pruebas ya existentes o la creación de otras nuevas.

En gran parte de población con discapacidad intelectual aparecen dificultades en habilidades básicas como lectura y escritura, memoria, orientación o funciones ejecutivas asociadas (Ke \& Liu, 2017). Asimismo las difi- 
cultades en la comunicación, aparecen según la edad, así entre un 15 y un 33 \% de las personas con DI en España (INE, 2018) muestran dificultades asociadas a estas áreas. El tiempo dedicado a la aplicación de los instrumentos de evaluación es fundamental e influye enormemente en el diseño de herramientas específicas. Factores como una mayor latencia de respuesta, dificultades en la atención y la concentración, mayor tiempo de espera en la ejecución de tareas, provocan que los procesos evaluativos mal diseñados resulten eternos y agotadores para personas que no suelen estar habituadas a este tipo de procedimientos. Además, la falta de baremos en población con discapacidad intelectual, supone que las comparaciones en los resultados obtenidos, dependan de la creación de una línea base del individuo, lo que requiere el uso de protocolos estandarizados. El personal técnico evaluador por tanto debe estar suficientemente formado en la aplicación de las pruebas y las peculiaridades propias de la comunicación con el cliente, además del uso de sistemas alternativos y/o aumentativos de la comunicación (Abril, Delgado \& Vigara, 2012).

El envejecimiento de las personas con discapacidad intelectual presenta ciertas particularidades que hay que tener presentes, entre ellas, según la Guía de Indicadores y Recomendaciones Personas con Discapacidad en proceso de envejecimiento (FEAPS, 2007), estarían que no existen enfermedades propias en las personas mayores, sino que estos tienen menor capacidad de recuperarse de ellas y enferman más a menudo, y además en la discapacidad intelectual, se da esta misma circunstancia, aunque es cierto que algunos síndromes y trastornos tienen comorbilidades asociadas relacionadas con enfermedades que aparecen en la vejez.

Las personas gravemente afectadas, y/o con daño cerebral pueden tener una menor esperanza de vida, y también sabemos que las personas con síndrome de Down, presentan un envejecimiento precoz y una alta prevalencia de aparición de deterioro cognitivo leve y enfermedad de Alzheimer (Esteba- Castillo, 2015). Debido a estas últimas consideraciones, es habitual establecer los protocolos de evaluación neuropsicológicos, emocionales y de salud mental, físicos y de salud a partir de los 45 años, que nos permitan establecer esa deseada línea base con la que poder establecer comparativas intra-sujeto (Alcedo, Aguado, Arias, González \& Rozada 2008).

Los factores sociales, relacionados con la oportunidad de tener una vida plena y en igualdad de condiciones y derechos están permitiendo el acceso a las PDI a servicios a los que no acudían tradicionalmente, algunos tan básicos como revisiones con médicos especialistas en audición, visión, medición interna, odontología, ginecología, etc. Mención especial debemos hacer al acceso de las personas con discapacidad intelectual a los servicios especializados en salud mental, dándose de forma muy habitual una polimedicación para resolver problemas de conducta a los que no se le ha dado respuesta con metodología preventiva no medicalizada (Flórez, 2005). Modelos de intervención como el apoyo conductual positivo, el apoyo activo, el enfoque centrado en la persona y la planificación basada en modelos de calidad de vida, están demostrando una importante eficacia y eficiencia en la intervención en estas personas (Navas, Verdugo, Martínez, Sainz \& Aza, 2019).

El objetivo de este estudio fue describir las características de una prueba de screening conductual de 31 ítems, diseñada para el registro de conductas asociadas al envejecimiento, y analizar su utilidad como prueba de detección de las conductas descritas en una muestra de personas con discapacidad intelectual en proceso de envejecimiento.

\section{METODOLOGÍA}

\section{Participantes}

La muestra de participantes estuvo conformada por 64 personas con discapacidad intelectual, con edades comprendidas entre 35 y 65 años, y con una media de edad de 48.38 (DT=7.02). De ellas 31 eran hombres (48.4\%) y 33 eran mujeres (51.6\%). Todos Ios participantes conviven con su familia en el domicilio familiar.

Con respecto a la asistencia a la escuela, el 1.6\% no ha asistido a la escuela, el $78.1 \%$ asistió a una escuela de educación especial, el 3.1\% asistió a una aula especial en una escuela ordinaria mientras que el 17.2\% asistió a una escuela ordinaria. Los participantes se seleccionaron entre los usuarios de la Asociación, que cumplieran con los criterios de edad y que dieran su consentimiento a participar en la investigación. En la Tabla 1 puede verse 
la distribución según sexo y el tipo de escuela a la que asistieron los participantes. La mayoría asistieron a una escuela de educación especial.

Tabla 1. Distribución de los participantes según el sexo y asistencia a la escuela. Frecuencia

\begin{tabular}{lccc}
\hline & Hombre & Mujer & Total \\
& $n$ & $n$ & $n$ \\
\hline No han asistido a la escuela & 1 & 0 & 1 \\
\hline Sí, a una escuela de educación especial & 26 & 24 & 05 \\
\hline Sí, a un aula especial en una escuela ordinaria & 1 & 1 & 2 \\
\hline Sí, a una escuela ordinaria & 3 & 8 & 11 \\
\hline
\end{tabular}

Con respecto a la distribución de los participantes según su ocupación, el 17.5\% asiste al Servicio de Terapia ocupacional y el $82.5 \%$ al Centro de atención especializada. En la Tabla 2 puede verse la distribución según el sexo y la ocupación. La mayoría de los participantes estaban asistiendo a un centro de atención especializada en el que reciben un tratamiento integral ajustado a sus necesidades.

Tabla 2. Distribución de los participantes según el sexo y ocupación principal. Frecuencia

\begin{tabular}{lccc}
\hline & Hombre & Mujer & Total \\
& $n$ & $n$ & $n$ \\
\hline Servicio de Terapia Ocupacional & 6 & 6 & 12 \\
\hline $\begin{array}{l}\text { Centro de atención especializada para personas con } \\
\text { necesidades de apoyo generalizadas }\end{array}$ & 25 & 27 & 52 \\
\hline
\end{tabular}

\section{Instrumentos}

Se recogieron datos sociodemográficos, como edad, sexo, si habían asistido a la escuela y el tipo de escuela, con quien convivían y la ocupación principal en el momento de la evaluación.

La Escala de Screening de Atención directa de Molina (2018) para personas con discapacidad intelectual. Es una Escala elaborada a partir de conductas de inventarios para personas mayores y está compuesta por 31 ítems que describen conductas observadas en personas en proceso de envejecimiento. Describe las conductas asociadas a los procesos psicológicos básicos, las actividades de la vida diaria (AVD) y el estado emocional, que aparecen más frecuentemente en personas en proceso de envejecimiento. Tiene un formato de respuesta dicotómica, indicando si se observa o no esa conducta y desde cuando se observa.

Para esta investigación se adaptó la escala de respuesta, así, tras la observación del sujeto, si se observa la conducta se registra según una escala de respuesta de cuatro categorías, que son 1 (no se observa la conducta); 2 (si se observa la conducta, pero no es un cambio conductual); 3 (si se observa la conducta, y es un cambio conductual de menos de 6 meses) y 4 (si se observa la conducta, y es un cambio conductual de más de 6 meses).

La escala es cumplimentada por parte de un profesional de atención directa, ya que necesita que el evaluador conozca a la persona a evaluar previamente a la observación y registro de su conducta. Las conductas hacen referencia a áreas fundamentales en la evaluación de las personas con discapacidad intelectual.

\section{Procedimiento}

Los participantes se seleccionaron entre las personas que pertenecen a una asociación que atiende a personas con discapacidad intelectual. La selección de la muestra se realizó mediante un muestreo intencional y restringido, ya que nos interesaba el grupo de personas con discapacidad en proceso de envejecimiento que asistían a los servicios de esa Asociación.

Se solicitó la aprobación del estudio a la junta de dirección de la Asociación, así como el consentimiento informado tanto a los participantes como a sus tutores. El estudio cumple con los requisitos de la Declaración de Helsinki, con todas las ampliaciones. 
La recogida de datos se realizó por parte de los profesionales de atención directa de la persona seleccionada, ya que la administración de la Escala requiere un conocimiento previo del sujeto a evaluar. La recogida de datos se realizó a lo largo de una semana para todos los participantes.

\section{Análisis de datos}

Los análisis de datos se realizaron con el paquete estadístico SPSS.24. Se realizaron análisis descriptivos, media y desviación típica para las variables cuantitativas, así como frecuencia y porcentajes para las variables categoriales. Se utilizó el análisis de correlación bivariada de Pearson, para analizar la relación entre la puntuación total de la Escala y la edad de los participantes.

Resultados

En la Tabla 3 pueden verse los resultados descriptivos de todos los ítems de la Escala de Screening de Atención directa (Molina, 2018). Según estos resultados hay ítems que podemos considerar que aportan una mayor información sobre los posibles cambios en las conductas de las personas evaluadas. Se considerarán como importantes aquellas conductas que muestren un porcentaje superior al $10 \%$ en respuestas en las opciones de sí ha habido un cambio, tanto inferior como superior a seis meses.

Según este criterio encontramos que las conductas en las que se detecta cambios son: anda más despacio, más torpe 0 arrastra los pies; le cuesta mantener la atención más que antes; se muestra más irritable; se le olvidan las consignas que se le han dado; está más dormido que antes; prefiere estar solo y tranquilo; se le olvida donde deja las cosas; ha tenido pérdidas (incontinencia); no se acuerda de los nombres (cosas, compañeros, familiares); dificultades para mostrar afectos, y sentimientos de dependencia.

Tabla 3. Estadísticos descriptivos, frecuencia y porcentaje, de las respuestas a los ítems de la Escala de Screening de Atención directa

\begin{tabular}{|c|c|c|c|c|}
\hline & No & $\begin{array}{l}\text { Sí, no es } \\
\text { un cambio }\end{array}$ & $\begin{array}{l}\text { Sí, cambio } \\
<6 \text { meses }\end{array}$ & $\begin{array}{l}\text { Sí, cambio } \\
>6 \text { meses }\end{array}$ \\
\hline Conductas Escala & $n(\%)$ & $n(\%)$ & $n(\%)$ & $n(\%)$ \\
\hline $\begin{array}{l}\text { 1. Anda más despacio, más torpe o } \\
\text { arrastra los pies }\end{array}$ & $43(67.2)$ & $5(7.8)$ & $6(9.4)$ & $10(15.6)$ \\
\hline $\begin{array}{l}\text { 2. Le cuesta mantener la atención } \\
\text { más que antes }\end{array}$ & $42(65.6)$ & $7(10.9)$ & $7(10.9)$ & $8(12.5)$ \\
\hline $\begin{array}{l}\text { 3. Le ha dado una crisis epiléptica } \\
\text { cuando no le había dado nunca }\end{array}$ & $62(96.9)$ & - & - & $2(3.1)$ \\
\hline $\begin{array}{l}\text { 4. Rechaza estar en el taller o en } \\
\text { ocio o en el área de apoyo }\end{array}$ & $59(92.2)$ & $3(4.7)$ & $1(1.6)$ & $1(1.6)$ \\
\hline 5. Se muestra más irritable & $47(73.4)$ & $1(1.6)$ & 12(18.8) & $4(6.3)$ \\
\hline $\begin{array}{l}\text { 6. Se le olvidan las consignas que } \\
\text { se le han dado }\end{array}$ & $51(79.9)$ & $2(3.1)$ & $8(12.5)$ & $3(4.7)$ \\
\hline 7. Han aparecido olvidos & $47(73.4)$ & $3(4.7)$ & $8(12.5)$ & $6(9.4)$ \\
\hline $\begin{array}{l}\text { 8. Se acerca mucho a la tarea. } \\
\text { Dificultades visuales }\end{array}$ & $50(78.1)$ & $8(12.5)$ & $2(3.1)$ & $4(6.3)$ \\
\hline $\begin{array}{l}\text { 9. Parece no escuchar. Dificultades } \\
\text { auditivas }\end{array}$ & $58(90.6)$ & $3(4.7)$ & $2(3.1)$ & $1(1.6)$ \\
\hline $\begin{array}{l}\text { 10. Llega tarde a su tarea cuando } \\
\text { antes era muy puntual }\end{array}$ & $61(95.3)$ & $2(3.1)$ & $1(1.6)$ & - \\
\hline 11. Está más dormido que antes & $45(70.3)$ & $3(4.7)$ & $10(15.6)$ & 6(9.4) \\
\hline
\end{tabular}




\begin{tabular}{|c|c|c|c|c|}
\hline $\begin{array}{l}\text { 12. Rechaza las actividades que } \\
\text { antes eran gratificantes }\end{array}$ & $58(90.6)$ & - & $2(3.1)$ & $4(6.3)$ \\
\hline 13. Prefiere estar solo y tranquilo & $39(60.9)$ & $14(21.9)$ & $4(6.3)$ & $7(10.9)$ \\
\hline 14. Rechaza el ruido y la música & $53(82.8)$ & $5(7.8)$ & $3(4.7)$ & $3(4.7)$ \\
\hline $\begin{array}{l}\text { 15. Se ha caído con frecuencia, } \\
\text { tropieza }\end{array}$ & $57(89.1)$ & $4(6.3)$ & $2(3.1)$ & $1(1.6)$ \\
\hline 16. Ha dejado de beber agua & $63(98.4)$ & $1(1.6)$ & - & - \\
\hline $\begin{array}{l}\text { 17. Se atraganta con frecuencia, } \\
\text { facilidad }\end{array}$ & $61(95.3)$ & - & $2(3.1)$ & $1(1.6)$ \\
\hline 18. Se le entiende menos al hablar & $60(93.8)$ & - & $3(4.7)$ & $1(1.6)$ \\
\hline 19. No responde a su nombre & $63(98.4)$ & - & & $1(1.6)$ \\
\hline $\begin{array}{l}\text { 20. Le cuesta lavarse las manos, } \\
\text { dientes, ducha }\end{array}$ & $55(85.9)$ & $5(7.8)$ & $1(1.6)$ & $3(4.7)$ \\
\hline $\begin{array}{l}\text { 21. Se le olvida donde deja las } \\
\text { cosas }\end{array}$ & $56(87.5)$ & $1(1.6)$ & $5(7.8)$ & 2(3.1) \\
\hline 22. Se desorienta por el taller & $61(95.3)$ & - & $2(3.1)$ & $1(1.6)$ \\
\hline $\begin{array}{l}\text { 23. Ha tenido pérdidas } \\
\text { (incontinencia) }\end{array}$ & $51(79.7)$ & $4(6.3)$ & $2(3.1)$ & $7(10.9)$ \\
\hline $\begin{array}{l}\text { 24. Siempre está con su bolso o con } \\
\text { su mochila cerca }\end{array}$ & $59(92.2)$ & - & $3(4.7)$ & $2(3.1)$ \\
\hline $\begin{array}{l}\text { 25. Rechaza salir a la calle } \\
\text { (salidas...) }\end{array}$ & $61(95.3)$ & $1(1.6)$ & $1(1.6)$ & $1(1.6)$ \\
\hline 26. Llora y ríe con facilidad & $48(75)$ & $10(15.6)$ & $4(6.3)$ & $2(3.1)$ \\
\hline $\begin{array}{l}\text { 27. No se acuerda de los nombres } \\
\text { (cosas, compañeros, familiares) }\end{array}$ & $52(81.3)$ & $4(6.3)$ & $5(7.8)$ & $3(4.7)$ \\
\hline $\begin{array}{l}\text { 28. Tiene o expresa sentimientos de } \\
\text { inutilidad }\end{array}$ & $58(90.6)$ & $3(4.7)$ & $1(1.6)$ & $2(3.1)$ \\
\hline $\begin{array}{l}\text { 29. Dificultades para mostrar } \\
\text { afectos }\end{array}$ & $55(85.9)$ & - & $8(12.5)$ & 1(1.6) \\
\hline 30. Sentimientos de dependencia & $50(78.1)$ & - & 12(18.8) & $2(3.1)$ \\
\hline $\begin{array}{l}\text { 31. Síntomas parecidos a los de una } \\
\text { infección de orina }\end{array}$ & $63(98.4)$ & $1(1.6)$ & & \\
\hline
\end{tabular}

No se observan cambios en las siguientes conductas de los participantes, o estos son muy poco frecuentes: Le ha dado una crisis epiléptica cuando no le había dado nunca; Ilega tarde a su tarea cuando antes era muy puntual; ha dejado de beber agua y síntomas parecidos a los de una infección de orina.

Se calculó la puntuación total de cambio conductual a partir del sumatorio de las puntuaciones obtenidas en todas las conductas, de manera que una mayor puntuación, supone un mayor cambio conductual. A partir de esta puntuación se calculó la correlación con la edad. Encontramos una correlación directa y estadísticamente significativa entre la edad y la puntuación total de cambio ( $r=0.309 ; p=.013)$.

Por último, se calculó las diferencias según el sexo en la puntuación total de cambio conductual, no encontramos diferencias estadísticamente significativas según el sexo. En la Tabla 4 pueden verse las puntuaciones medias, y podemos observar que la puntuación media en las mujeres es superior a los hombres, aunque las diferencias no son estadísticamente significativas. 
Tabla 4. Diferencias según el sexo en la puntuación total de cambio conductual

\begin{tabular}{|c|c|c|c|c|c|c|}
\hline Sexo & $n$ & $M$ & $D T$ & $\begin{array}{c}\text { Media de } \\
\text { error } \\
\text { estándar }\end{array}$ & $t$ & $p$ \\
\hline Hombre & 31 & 38.03 & 7.319 & 1.315 & -1.709 & .092 \\
\hline Mujer & 33 & 41.61 & 9.230 & 1.607 & & \\
\hline
\end{tabular}

\section{DISCUSIÓN Y CONCLUSIONES}

El objetivo de este estudio fue describir las características de una prueba de screening conductual, diseñada para el registro de conductas asociadas al envejecimiento, y analizar su utilidad como prueba de detección de las conductas descritas en una muestra de personas con discapacidad intelectual en proceso de envejecimiento. Para ello se administró a una muestra de personas con discapacidad intelectual, y se calculó la frecuencia de cada conducta, y la puntuación total en cambio conductual.

Los resultados ponen de manifiesto la presencia de cambios conductuales en el momento de la recogida de datos en las personas participantes, según son indicados por el personal que les atiende. Los cambios más frecuentes se observan en conductas relacionadas con el cansancio físico, así como con la memoria y con síntomas afectivos. No se observan modificaciones en conductas relacionadas con cambios somáticos. Es importante destacar, que este screening forma parte de un protocolo más complejo, multidisciplinar, en el que otras variables son estudiadas con mayor amplitud y profundidad, como el estudio de las variables físicas, médicas, prácticas y neuropsicológicas. El fin último de la prueba es aportar la visión de las personas de referencia (aquellas que tiene un contacto más constante con las personas estudiadas) en cuanto a las modificaciones en la conducta, de una forma breve.

En todo caso, aunque los síntomas de envejecimiento aparecen de forma prematura (la media de edad de la muestra es de 48.38 años), los resultados revelan un envejecimiento muy similar al de la población general, en el cual, suele aparecer un declive progresivo en el funcionamiento de algunas áreas cognitivas como la memoria 0 la atención (Barrera, \& Donolo, 2009), psicológicas como la dependencia y la irritabilidad y físicas como la pérdida de energía o el enlentecimiento motor, inherentes al paso de los años y la pérdida de habilidades y autonomía (IMSERSO, 2009). No obstante siempre debemos tener en cuenta, si las conductas observadas relacionadas con las dificultades visuales y auditivas así como con la epilepsia, están ya tratadas en los participantes en el momento de la recogida de datos.

López Mongil (2017), investigó la utilidad del estudio funcional de la conducta del individuo, por su relación con el deterioro cognitivo leve. Para ello utilizó entre otros el Test Mongil de AAVD (López Mongil, López Trigo, \& Gordaliza Ramos, 2013). Encontró que estas herramientas son útiles para identificar y monitorizar el estado del paciente, y que además son sencillas y breves, lo que facilita la generalización de su uso. Resulta interesante tener en cuenta sus conclusiones acerca de la utilización de estos resultados como objetivo terapéutico, en nuestro protocolo, los resultados obtenidos en la evaluación influyen directamente en la intervención en el centro al que acuden y la planificación de los apoyos y la atención individualizada recibida (López Mongil, 2017).

Estudios recientes, han demostrado que el estado de salud en el que los individuos llegan a los primeros años de la vejez y el cuidado en estos años, relativo a los hábitos de vida saludable (ejercicio físico, alimentación, estimulación cognitiva e higiene de sueño) así como sus relaciones interpersonales y otros factores sociales y económicos, determinan enormemente la aparición de signos prematuros de envejecimiento, frente a lo que hoy se denomina envejecimiento activo y de éxito o saludable (Petretto, Pili, Gaviano, López \& Zuddas, 2016). Pensamos que al igual que se ha producido un aumento en la esperanza de vida en las PDI mediante la mejora de la atención a este colectivo y su participación plena en la sociedad, es probable que la calidad de sus últimos años mejore también si se mejora la atención a personas mayores con discapacidad intelectual. Para ello, es necesario que los recursos asociados sean especializados para ese grupo de edad, que se atiendan a los mismos aspectos que predicen una buena calidad de vida en población general. 


\section{EVALUACIÓN DEL ENVEJECIMIENTO EN PERSONAS CON DISCAPACIDAD INTELECTUAL MEDIANTE UNA PRUEBA DE SCREENING CONDUCTUAL}

Consideración a parte en el estudio, tendrían aquellos casos en los que se detectase un deterioro patológico. En este caso los signos encontrados deberían ser comparados con otra muestra de personas con envejecimiento patológico en población sin discapacidad intelectual.

Como hemos descrito, las personas adultas con DI experimentan un mayor riesgo de desarrollar problemas de salud crónicos, sin embargo la inclusión de estos en programas de prevención de la salud, parecen estar homogeneizando los signos de sintomatología en los últimos años de la vida, así como mejorando el estado de salud en el que llegan a ellos. Consideramos que la Escala de Screening de Atención directa (Molina, 2018) es una prueba sencilla y rápida de aplicación y es sensible a los cambios conductuales que tienen lugar en el envejecimiento en personas con discapacidad intelectual. Este instrumento es útil también para que personas cuidadoras sin formación en salud, puedan detectar la aparición de posibles síntomas de un envejecimiento patológico, especialmente para aquellas que apoyan a personas con grandes necesidades de apoyo y que puedan tener dificultades para detectar 0 expresar indicios propios.

No obstante es necesaria más investigación que proporcione las puntuaciones de corte, para poder detectar tempranamente un deterioro patológico asociado al envejecimiento. Para ello es necesario continuar con la investigación, ampliando la muestra de participantes, además de estableciendo comparaciones con participantes sin discapacidad intelectual en proceso de envejecimiento.

El estudio cuenta con limitaciones, como el tamaño de la muestra de participantes y el método de muestreo, que no permiten la generalización de los resultados. También sería necesario contar con otros observadores que permitieran la comparación de la observación, así como otros instrumentos que permitieran la realización de estudios de validez convergente. Esperamos en futuras investigaciones poder dar respuesta a alguna de ellas. Pese a todo, creemos que las aportaciones son interesantes, ya que es necesario disponer de medidas sencillas y rápidas de aplicar que sean sensibles a los cambios conductuales en un mismo sujeto y que permitan a profesionales, cuidadores y familiares detectar cambios y así intervenir precozmente.

\section{REFERENCIAS BIBLIOGRÁFICAS}

Abril Abadín, D., Delgado Santos, C. I., \& Vigara Cerrato, Á. (2012). Comunicación aumentativa y alternativa. Guía de referencia. CEAPAT.

Alcedo Rodríguez, M., Aguado Díaz, A. L., Arias Martínez, B., González González, M., \& Rozada Rodríguez, C. (2008). Escala de Calidad de Vida (ECV) para personas con discapacidad que envejecen: Estudio preliminar. Psychosocial Intervention, 17(2), 153-167.

American Psychological Association, APA (2013). Manual Estadístico y Diagnóstico de los Trastornos mentales DSM-5 (5E․). Masson.

Barrera, M. L., \& Donolo, D. (2009) Diagnóstico diferencial de envejecimiento patológico. Desempeños en el Addenbrooke's Cognitive Examination. Universidad Nacional de Río Cuarto- Córdoba (Argentina)

Del Campo, J. B., Arias, M., Fernández, M. I. R., \& Castro, F. V. (2007). Envejecimiento y discapacidad intelectual; la nueva etapa. International Journal of Developmental and Educational Psychology, 1(2), 43-56.

Esteba-Castillo, S. (2015). Neuropsicología de la discapacidad intelectual con y sin origen genético. Universidad Autónoma de Barcelona.

FEAPS Aragón (2007). Guía de Indicadores y Recomendaciones Personas con Discapacidad en proceso de envejecimiento. Aragón.

Flórez, J. (2005). La salud de las personas con discapacidad. Revista Síndrome de Down, 22, 8-14.

García-Alba, J., Esteba-Castillo, S., Viñas-Jornet, M. (2018). Neuropsicología de la discapacidad intelectual de origen genético. Editorial Síntesis. Madrid

Instituto de Mayores y Servicios Sociales, IMSERSO (2009). Las personas mayores en España. Informe Ministerio de Sanidad y Política Social. Ed. Madrid. 
Instituto Nacional de Estadística, INE (2018). Encuesta de Discapacidad, Autonomía Personal y situaciones de Dependencia. Consultado en https://www.observatoriodeladiscapacidad.info/discapacidad-autonomia-personal-y-situaciones-de-dependencia-ine/

Ke, X., \& Liu, J. (2017). Discapacidad intelectual. Traducción de Irarrázaval, M., Martin, A., Prieto-Tagle, F. y Fuertes, 0. En Rey, Joseph. Manual de Salud Mental Infantil y Adolescente de la IACAPAP, 1-28.

López Mongil, R. (2017). Deterioro cognitivo/demencia: valoración y seguimiento. Aspectos funcionales. Revista Española de Geriatría y Gerontología, 52, 28-33.

López Mongil, R., López Trigo, J.A. \& Gordaliza Ramos, A. (2013). El Test Mongil de actividades de la vida diaria básicas, instrumentales y avanzadas y su utilidad en el envejecimiento. International Journal of Developmental and Educational Psychology, 2, 221-226.

Molina, L. (2018). Escala de Screening de Atención directa de Molina. Documento no publicado.

Navas Macho, P., Verdugo Alonso, M. Á., Martínez Torres, S., Sainz Modinos, F., \& Aza Hernández, A. (2019). Derechos y calidad de vida en personas con discapacidad intelectual y mayores necesidades de apoyo. Siglo Cero, 48, 7-66.

Petretto, D. R., Pili, R., Gaviano, L., López, C. M., \& Zuddas, C. (2016). Envejecimiento activo y de éxito o saludable: una breve historia de modelos conceptuales. Revista Española de Geriatría y Gerontología, 51(4), 229241. 
